DOI https://doi.org/10.30525/978-9934-26-019-3-2

\title{
FINITE ELEMENT MODELING OF THE STRESS-STRAIN STATE OF A COMPOSITE MATERIAL WITH A VISCOELASTIC MATRIX
}

\section{Grebenyuk S. M., Klymenko M. I.}

\section{INTRODUCTION}

Due to their unique properties, fibrous composite materials are widely used in the creation of new equipment, structures, etc. The materials of which the composite is produced have significant differences in their properties. Accordingly, a composite material with a viscoelastic matrix and transtropic fiber is often used. Developing a mathematical model that would take into account these properties is a rather complex procedure. As a rule, further study of the developed model is impossible without the use of numerical methods, such as the finite element method (FEM). But the use of FEM to solve the problem of viscoelasticity of the fibrous composite requires improvement and modification of the method to take into account the peculiarities of the developed model.

A significant amount of works is devoted to research in this area. In [1], a finite element analysis of the effect of viscoelastic properties of the matrix on the stress-strain state of an epoxy beam reinforced with fibers was performed. Studies of residual stresses in glass-epoxy material having orthotropic viscoelastic properties are presented in [2] using the finite element method. The study of bound bodies of complex geometry with different degrees of aging was carried out using the combined perturbation finite element-boundary element method [3]. A space-time finite element has been developed for the study of viscoelastic deformation of fibrous composites, which is described by difference kernels with instantaneous and long-term mechanical characteristics [4]. In [5] the special grid homogenization method by the method of finite elements of viscoelastic composite material is presented.

\section{Homogenization of a composite material}

We will assume that the composite material consists of two components: an isotropic viscoelastic matrix and a transtropic elastic fiber. In this case, the mechanical characteristics of the components will 
be as follows. For transtrop elastic fiber: $E_{1}^{\circ}, E_{2}^{\circ}$ are longitudinal and transverse modulus of elasticity of the first kind for the fiber (index 1 corresponds to the direction of the axis of the fiber, directions 2 and 3 determine the plane of isotropy, i.e. $\left.E_{3}^{\circ}=E_{2}^{\circ}\right) ; v_{i j}^{\circ}$ mean Poisson's ratios $(i, j=1,2,3)$, whereby $v_{12}^{\circ}=v_{13}^{\circ}$ the condition $v_{i j}^{\circ} E_{j}^{\circ}=v_{j i}^{\circ} E_{i}^{\circ}$ is fulfilled; $G_{i j}^{\circ}$ mean shear modules $(i, j=1,2,3)$, whereby $G_{12}^{\circ}=G_{13}^{\circ}$ and $G_{i j}^{\circ}=G_{j i}^{\circ}$. For an isotropic viscoelastic matrix: $E^{*}$ is elastic modulus, $v^{*}$ is Poisson's ratio, the shear modulus is determined by the formula $G^{*}=E^{*} /\left(2\left(1+v^{*}\right)\right)$. To model the viscoelastic properties of the matrix, we use the Boltzmann-Volterra hereditary theory and replace the elastic modulus with an integral operator of the form:

$$
\tilde{E}^{*}[\varepsilon(t)]=E^{*}\left(\varepsilon(t)-\int_{0}^{t} R^{*}(t-\tau) \varepsilon(\tau) d \tau\right)
$$

where $R^{*}(t-\tau)$ is the difference relaxation kernel of the matrix material, $E^{*}$ is the instantaneous value of the elastic modulus of the matrix material.

The composite material will be represented by a homogeneous transtropic viscoelastic material, the mechanical properties of which depend on the mechanical properties of the matrix and fiber materials, and the volume fraction of each of them in the composite. The mechanical properties of transtropic material are described by five independent variables. When modeling the viscoelastic behavior of a composite material, some of these variables are integral operators, similar in structure to (1). Therefore, for the viscoelastic transtropic material it is necessary to determine five instantaneous variables of constants and five relaxation functions. By accepting some hypotheses, the number of required characteristics can be reduced. Accordingly, in [6], assuming that the Poisson's ratios are constant over time, the mechanical integral characteristics of the viscoelastic transtropic material modeling the composite were found by the method of a representative volume element. In the reinforcement coordinate system, the stress-strain state of the material will be determined by the relations: 


$$
\begin{gathered}
\tilde{\sigma}^{11}=\tilde{C}^{1111}\left[\varepsilon_{11}\right]+\tilde{C}^{1122}\left[\varepsilon_{22}\right]+\tilde{C}^{1122}\left[\varepsilon_{33}\right] ; \\
\tilde{\sigma}^{22}=\tilde{C}^{122}\left[\varepsilon_{11}\right]+\tilde{C}^{2222}\left[\varepsilon_{22}\right]+\tilde{C}^{2233}\left[\varepsilon_{33}\right] ; \\
\tilde{\sigma}^{33}=\tilde{C}^{1122}\left[\varepsilon_{11}\right]+\tilde{C}^{2233}\left[\varepsilon_{22}\right]+\tilde{C}^{2222}\left[\varepsilon_{33}\right] ; \\
\tilde{\sigma}^{12}=\tilde{C}^{1212} \varepsilon_{12} ; \\
\tilde{\sigma}^{13}=\tilde{C}^{1212} \varepsilon_{13} ; \\
\tilde{\sigma}^{23}=\tilde{C}^{2323} \varepsilon_{23},
\end{gathered}
$$

here the integral operator has the following form:

$$
\tilde{C}^{i j k l}[\varphi(t)]=C^{i j k l}\left(\varphi(t)-\int_{0}^{t} K^{(i j k l)}(t-\tau) \varphi(\tau) d \tau\right)
$$

where $\varphi(t)$ is the function on which the integrated operator acts, $K^{(j i k l)}(t-\tau)$ is the difference relaxation kernel of mechanical characteristics $\tilde{C}^{i j k}$.

Integral operators that reflect the viscoelastic properties of the transtropic composite can be written as follows:

$$
\begin{gathered}
\tilde{C}^{1111}[\varphi]=\frac{\tilde{E}_{1}[\varphi]\left(1-v_{23}^{2}\right)}{1-2 v_{12} v_{21}-v_{23}^{2}-2 v_{12} v_{21} v_{23}} ; \\
\tilde{C}^{1122}[\varphi]=\frac{\tilde{E}_{1}[\varphi] v_{21}\left(1+v_{23}\right)}{1-2 v_{12} v_{21}-v_{23}^{2}-2 v_{12} v_{21} v_{23}} ; \\
\tilde{C}^{2222}[\varphi]=\frac{\tilde{E}_{1}[\varphi] v_{21}\left(1-v_{12} v_{21}\right)}{v_{12}\left(1-2 v_{12} v_{21}-v_{23}^{2}-2 v_{12} v_{21} v_{23}\right)} ; \\
\tilde{C}^{2233}[\varphi]=\frac{\tilde{E}_{1}[\varphi] v_{21}\left(v_{23}+v_{12} v_{21}\right)}{v_{12}\left(1-2 v_{12} v_{21}-v_{23}^{2}-2 v_{12} v_{21} v_{23}\right)} ; \\
\tilde{C}^{3333}[\varphi]=\frac{\tilde{E}_{1}[\varphi] v_{21}\left(1+v_{23}\right)}{1-2 v_{12} v_{21}-v_{23}^{2}-2 v_{12} v_{21} v_{23}} ;
\end{gathered}
$$




$$
\begin{gathered}
\tilde{C}^{1212}[\varphi]=2 \tilde{G}_{12}[\varphi] ; \\
\tilde{C}^{2323}[\varphi]=\frac{\tilde{E}_{1}[\varphi] v_{21}}{v_{12}\left(1+v_{23}\right)} .
\end{gathered}
$$

Expressions for the effective values of the constant Poisson's ratios are:

$$
\begin{gathered}
v_{12}=\frac{\left(\alpha-2 \beta v_{12}^{\circ}\right) v^{*}+2 f E_{2}^{\circ}\left(v_{12}^{\circ}-v^{*}\right)}{\alpha-2 \beta v_{12}^{\circ}+2 f v^{*} E_{2}^{\circ}\left(v_{12}^{\circ}-v^{*}\right)}, \\
v_{21}=v_{12} \frac{E_{2}}{E_{1}}, \\
v_{23}=\frac{\alpha\left(\left(1+v^{*}\right)+4\left(\gamma-2 E^{*}\right)\left(1-\left(v^{*}\right)^{2}\right)\left(f \chi_{1}+\chi_{2}\right)\right)-\left(\delta+\eta_{1} \eta_{2}\right)}{\alpha\left(\left(1+v^{*}\right)+4\left(\gamma-2 E^{*}\right)\left(1-\left(v^{*}\right)^{2}\right)\left(f \chi_{1}+\chi_{2}\right)\right)+\left(\delta+\eta_{1} \eta_{2}\right)}
\end{gathered}
$$

or

$$
v_{23}=\frac{\alpha\left(\left(1+v^{*}\right)+4\left(\gamma-2 E^{*}\right)\left(1-\left(v^{*}\right)^{2}\right)\left(f \chi_{1}-\chi_{2}\right)\right)-\left(\delta+\eta_{1} \eta_{2}\right)}{\alpha\left(\left(1+v^{*}\right)+4\left(\gamma-2 E^{*}\right)\left(1-\left(v^{*}\right)^{2}\right)\left(f \chi_{1}-\chi_{2}\right)\right)+\left(\delta+\eta_{1} \eta_{2}\right)},
$$

where $f$ is the volume fraction of fiber in the composite material,

$$
\begin{gathered}
\alpha=E^{*}(1-f)\left(1-v_{23}^{\circ}\right)+E_{2}^{\circ}\left(f\left(1-v^{*}\right)+\left(1+v^{*}\right)\right) ; \\
\beta=v_{21}^{\circ} E^{*}(1-f)+v^{*} f E_{2}^{\circ} ; \\
\delta=f\left(1+v^{*}\right)\left(\gamma-2 E_{2}^{\circ}\right)+\gamma\left(1-v^{*}\right) ; \\
\eta_{1}=2 E_{1}^{\circ} E^{*} f(f-1)\left(\gamma v^{*}-2 v_{21}^{\circ} E^{*}\right)^{2} ; \\
\frac{1}{E^{*}\left(\alpha\left(f E_{1}^{\circ}+E^{*}(1-f)\right)-2 \beta\left(f v^{*} E_{1}^{\circ}+v_{12}^{\circ} E^{*}(1-f)\right)\right)} \\
\gamma=E^{*}\left(1-v_{23}^{\circ}\right)+E_{2}^{\circ}\left(1+v^{*}\right) ; \\
\chi_{1}=\frac{d_{11}-d_{21}}{d_{11} d_{22}-d_{21} d_{12}} ;
\end{gathered}
$$




$$
\begin{gathered}
\chi_{2}=\frac{d_{22}-d_{12}}{d_{11} d_{22}-d_{21} d_{12}} ; \\
d_{11}=E_{2}^{\circ}\left(1+v^{*}\right)\left(4 f v^{*}-3-\frac{1}{f^{2}}\right)- \\
-E^{*}\left(\left(4 f-\frac{1}{f^{2}}-3\right)\left(1+v_{23}^{\circ}\right)+4\left(\frac{1}{f^{2}}-f\right)\left(1-v_{21}^{\circ} v_{12}^{\circ}\right)\right) ; \\
d_{12}=E_{2}^{\circ}\left(1+v^{*}\right)\left(4 v^{*}-4+\frac{1}{f}-f\right)- \\
-E^{*}\left(\left(\frac{1}{f}-f\right)\left(1+v_{23}^{\circ}\right)+4\left(1-\frac{1}{f}\right)\left(1-v_{21}^{\circ} v_{12}^{\circ}\right)\right) ; \\
d_{21}=E_{2}^{\circ}\left(1+v^{*}\right)\left(6 f-4 f v^{*}+\frac{1}{f^{2}}-3\right)- \\
-E^{*}\left(\left(2 f+\frac{1}{f^{2}}-3\right)\left(1+v_{23}^{\circ}\right)+4\left(f-\frac{1}{f^{2}}\right)\left(1-v_{21}^{\circ} v_{12}^{\circ}\right)\right) ; \\
d_{22}=E_{2}^{\circ}\left(1+v^{*}\right)\left(4 v^{*}-2-f-\frac{1}{f}\right)- \\
-E^{*}\left(\left(2-f-\frac{1}{f}\right)\left(1+v_{23}^{\circ}\right)+4\left(\frac{1}{f}-1\right)\left(1-v_{21}^{\circ} v_{12}^{\circ}\right)\right) .
\end{gathered}
$$

The instantaneous value of the effective transverse elastic modulus is determined by the formulas:

$$
E_{2}=\frac{2 \alpha E^{*}}{\alpha\left(\left(1+v^{*}\right)+4\left(\gamma-2 E^{*}\right)\left(1-\left(v^{*}\right)^{2}\right)\left(f \chi_{1}+\chi_{2}\right)\right)+\left(\delta+\eta_{1} \eta_{2}\right)}
$$

or

$$
E_{2}=\frac{2 \alpha E^{*}}{\alpha\left(\left(1+v^{*}\right)+4\left(\gamma-2 E^{*}\right)\left(1-\left(v^{*}\right)^{2}\right)\left(f \chi_{1}-\chi_{2}\right)\right)+\left(\delta+\eta_{1} \eta_{2}\right)} .
$$

The two integral effective characteristics have a form similar to (1), and contain two components - the instantaneous value and the relaxation kernel: 


$$
\begin{aligned}
& \tilde{E}_{1}[\varepsilon(t)]=E_{1}\left(\varepsilon(t)-\int_{0}^{t} R_{E}(t-\tau) \varepsilon(\tau) d \tau\right), \\
& \tilde{G}_{12}[\varepsilon(t)]=G_{12}\left(\varepsilon(t)-\int_{0}^{t} R_{G}(t-\tau) \varepsilon(\tau) d \tau\right),
\end{aligned}
$$

where $E_{1}$ is the instantaneous value of the longitudinal elastic modulus of the first kind of homogeneous transtropic material, $G_{12}$ is the instantaneous value of the longitudinal shear modulus of the homogeneous transtropic material, $R_{E}(t-\tau)$ is the difference relaxation kernel of the longitudinal elastic modulus of the first kind of homogeneous transtropic material, $R_{G}(t-\tau)$ is the difference relaxation kernel of the longitudinal shear modulus of homogeneous transtropic material.

It is a difficult task to find these characteristics because of the structure of the composite material, which is heterogeneous and is made of two components. The properties of the components differ from each other, so they use different mathematical formalization. In this case, effective mechanical characteristics are found by the Laplace transform. The instantaneous value of the effective longitudinal elastic modulus of the first kind is as follows:

$$
E_{1}=\frac{f+(1-f) k}{\alpha_{1}+\alpha_{2}\left(k_{6}-k_{5} E^{*}-k_{7} k\right)},
$$

Where

$$
\begin{gathered}
\alpha_{1}=\frac{1-v_{23}^{\circ}-2 v_{12}^{\circ} v_{21}^{\circ}}{E_{1}^{\circ}\left(1-v_{23}^{\circ}\right)}, \alpha_{2}=\frac{2 v_{21}^{\circ}}{1-v_{23}^{\circ},} \\
\beta_{1}=\frac{\left(1+v^{*}\right)\left(1-2 v^{*}\right)}{\left(1-v^{*}\right)}, \beta_{2}=\frac{2 v^{*}}{1-v^{*}}, \\
\gamma_{1}=\frac{E_{2}^{\circ} v_{12}^{\circ}}{E_{1}^{\circ}\left(1-v_{23}^{\circ}\right)}, \gamma_{2}=\frac{E_{2}^{\circ}}{1-v_{23}^{\circ},} \\
m_{0}=\frac{1}{\left(\alpha_{2}-\beta_{2}\right)\left(1-v^{*}\right) f+\alpha_{2}\left(1+v^{*}\right)}, \\
k_{1}=m_{0} \alpha_{1}\left(1-v^{*}\right) f, k_{2}=m_{0}\left(\alpha_{2} v^{*}\left(1+v^{*}\right)+\beta_{1}\left(1-v^{*}\right) f\right), \\
k_{3}=m_{0} \alpha_{1}\left(1+v^{*}\right), k_{4}=m_{0}\left(1+v^{*}\right)\left(v^{*}\left(\alpha_{2}-\beta_{2}\right)-\beta_{1}\right),
\end{gathered}
$$




$$
\begin{gathered}
k_{5}=\frac{m_{0} \alpha_{1}(f-1)}{\gamma_{2}}, k_{6}=\frac{\gamma_{1}}{\gamma_{2}}, \\
k_{7}=\frac{1}{\gamma_{2}}\left(\frac{v^{*}}{1-v^{*}}-m_{0}\left(\frac{\alpha_{2} v^{*}\left(1+v^{*}\right)}{1-v^{*}}+v^{*}\left(\alpha_{2}-\beta_{2}\right)-\beta_{1}(f-1)\right)\right), \\
k=\frac{E^{*}\left(k_{1}+k_{3}+k_{6}-k_{5} E^{*}\right)}{k_{7} E^{*}+k_{2}-k_{4}} .
\end{gathered}
$$

Presentation of the relaxation kernel for the longitudinal elastic modulus of a homogeneous material:

$$
R_{E}(p)=\frac{E_{1} F_{2}(p)-F_{1}(p)}{E_{1} F_{2}(p)},
$$

where

$$
\begin{gathered}
F_{1}(p)=f\left(k_{7} E^{*}\left(1-\tilde{R}^{*}(p)\right)+k_{2}-k_{4}\right)+ \\
+(1-f) E^{*}\left(k_{1}+k_{3}+k_{6}-k_{5} E^{*}\left(1-\tilde{R}^{*}(p)\right)\right)\left(1-\tilde{R}^{*}(p)\right), \\
F_{2}(p)=\left(\left(\alpha_{1}+\alpha_{2} k_{6}\right)\right)\left(k_{7} E^{*}\left(1-\tilde{R}^{*}(p)\right)+k_{2}-k_{4}\right)- \\
-\alpha_{2} k_{5} E^{*}\left(k_{7} E^{*}\left(1-\tilde{R}^{*}(p)\right)+k_{2}-k_{4}\right)\left(1-\tilde{R}^{*}(p)\right),
\end{gathered}
$$

where $\tilde{R}^{*}(p)$ is the presentation of the relaxation kernel for the matrix material.

The instantaneous value of the effective longitudinal shear modulus is written as:

$$
G_{12}=\frac{G^{*}\left(G_{12}^{\circ}(1+f)+G^{*}(1-f)\right)}{G_{12}^{\circ}(1-f)+G^{*}(1+f)} .
$$

Presentation of the relaxation kernel for the longitudinal shear modulus of a homogeneous material: 


$$
R_{G}(p)=\frac{C_{1}\left(1-\tilde{R}^{*}(p)\right)^{2}+C_{2}\left(1-\tilde{R}^{*}(p)\right)+C_{3}}{C_{4}\left(\left(1-\tilde{R}^{*}(p)\right)+C_{5}\right)},
$$

where

$$
\begin{gathered}
C_{1}=(f-1)\left(G^{*}\right)^{2}, C_{2}=G^{*}(1+f)\left(G_{12}-G_{12}^{\circ}\right), \\
C_{3}=G_{12} G_{12}^{\circ}(1-f), C_{4}=G_{12} G^{*}(1+f), \\
C_{5}=\frac{G_{12}^{\circ}(1-f)}{G^{*}(1+f)} .
\end{gathered}
$$

\section{Obtaining the law of the state}

For further practical use, it is necessary to determine their originals from the obtained presentations of relaxation kernels. The complexity of such an inverse transformation depends significantly on the type of relaxation matrix kernel. After finding the originals of the relaxation kernel taking into account (4)-(15) the action of integral operators (3) on some deformation function $\varepsilon$ can be described by dependencies:

$$
\begin{gathered}
\tilde{C}^{1111}[\varepsilon]=\frac{E_{1}\left(1-v_{23}^{2}\right)}{1-2 v_{12} v_{21}-v_{23}^{2}-2 v_{12} v_{21} v_{23}}\left(\varepsilon(t)-\int_{0}^{t} R_{E}(t-\tau) \varepsilon(\tau) d \tau\right) ; \\
\tilde{C}^{1122}[\varepsilon]=\frac{E_{1} v_{21}\left(1+v_{23}\right)}{1-2 v_{12} v_{21}-v_{23}^{2}-2 v_{12} v_{21} v_{23}}\left(\varepsilon(t)-\int_{0}^{t} R_{E}(t-\tau) \varepsilon(\tau) d \tau\right) ; \\
\tilde{C}^{2222}[\varepsilon]=\frac{E_{1} v_{21}\left(1-v_{12} v_{21}\right)}{v_{12}\left(1-2 v_{12} v_{21}-v_{23}^{2}-2 v_{12} v_{21} v_{23}\right)}\left(\varepsilon(t)-\int_{0}^{t} R_{E}(t-\tau) \varepsilon(\tau) d \tau\right) ; \\
\tilde{C}^{2233}[\varepsilon]=\frac{E_{1} v_{21}\left(v_{23}+v_{12} v_{21}\right)}{v_{12}\left(1-2 v_{12} v_{21}-v_{23}^{2}-2 v_{12} v_{21} v_{23}\right)}\left(\varepsilon(t)-\int_{0}^{t} R_{E}(t-\tau) \varepsilon(\tau) d \tau\right) ; \\
\tilde{C}^{3333}[\varepsilon]=\frac{E_{1} v_{21}\left(1+v_{23}\right)}{1-2 v_{12} v_{21}-v_{23}^{2}-2 v_{12} v_{21} v_{23}}\left(\varepsilon(t)-\int_{0}^{t} R_{E}(t-\tau) \varepsilon(\tau) d \tau\right) ; \\
\tilde{C}^{1212}[\varepsilon]=2 G_{12}\left(\left(\varepsilon(t)-\int_{0}^{t} R_{G}(t-\tau) \varepsilon(\tau) d \tau\right)\right) ; \\
\tilde{C}^{2323}[\varepsilon]=\frac{E_{1} v_{21}}{v_{12}\left(1+v_{23}\right)}\left(\varepsilon(t)-\int_{0}^{t} R_{E}(t-\tau) \varepsilon(\tau) d \tau\right) .
\end{gathered}
$$


In the right-hand sides of these equations we distinguish constant multipliers:

$$
\begin{gathered}
C_{0}^{1111}=\frac{E_{1}\left(1-v_{23}^{2}\right)}{1-2 v_{12} v_{21}-v_{23}^{2}-2 v_{12} v_{21} v_{23}} ; \\
C_{0}^{1122}=\frac{E_{1} v_{21}\left(1+v_{23}\right)}{1-2 v_{12} v_{21}-v_{23}^{2}-2 v_{12} v_{21} v_{23}} ; \\
C_{0}^{2222}=\frac{E_{1} v_{21}\left(1-v_{12} v_{21}\right)}{v_{12}\left(1-2 v_{12} v_{21}-v_{23}^{2}-2 v_{12} v_{21} v_{23}\right)} ; \\
C_{0}^{2233}=\frac{E_{1} v_{21}\left(v_{23}+v_{12} v_{21}\right)}{v_{12}\left(1-2 v_{12} v_{21}-v_{23}^{2}-2 v_{12} v_{21} v_{23}\right)} ; \\
C_{0}^{3333}=\frac{E_{121} v_{21}\left(1+v_{23}\right)}{1-2 v_{12} v_{21}-v_{23}^{2}-2 v_{12} v_{21} v_{23}} ; \\
C_{0}^{1212}=2 G_{12} ; \\
C_{0}^{2323}=\frac{E_{1} v_{21}}{v_{12}\left(1+v_{23}\right)} .
\end{gathered}
$$

Then the law of state in the presentations takes the form:

$$
\begin{aligned}
& \tilde{\sigma}^{11}=C_{0}^{1111}\left(\varepsilon_{11}(t)-\int_{0}^{t} R_{E}(t-\tau) \varepsilon_{11}(\tau) d \tau\right)+ \\
&+C_{0}^{1122}\left(\varepsilon_{22}(t)-\int_{0}^{t} R_{E}(t-\tau) \varepsilon_{22}(\tau) d \tau\right)+ \\
&+C_{0}^{1122}\left(\varepsilon_{33}(t)-\int_{0}^{t} R_{E}(t-\tau) \varepsilon_{33}(\tau) d \tau\right) \\
& \tilde{\sigma}^{22}=C_{0}^{1122}\left(\varepsilon_{11}(t)-\int_{0}^{t} R_{E}(t-\tau) \varepsilon_{11}(\tau) d \tau\right)+ \\
&+C_{0}^{2222}\left(\varepsilon_{22}(t)-\int_{0}^{t} R_{E}(t-\tau) \varepsilon_{22}(\tau) d \tau\right)+ \\
&+C_{0}^{2233}\left(\varepsilon_{33}(t)-\int_{0}^{t} R_{E}(t-\tau) \varepsilon_{33}(\tau) d \tau\right) \\
& \tilde{\sigma}^{33}=C_{0}^{1122}\left(\varepsilon_{11}(t)-\int_{0}^{t} R_{E}(t-\tau) \varepsilon_{11}(\tau) d \tau\right)+
\end{aligned}
$$




$$
\begin{aligned}
& +C_{0}^{2233}\left(\varepsilon_{22}(t)-\int_{0}^{t} R_{E}(t-\tau) \varepsilon_{22}(\tau) d \tau\right)+ \\
& +C_{0}^{2222}\left(\varepsilon_{33}(t)-\int_{0}^{t} R_{E}(t-\tau) \varepsilon_{33}(\tau) d \tau\right) ; \\
& \tilde{\sigma}^{12}=C_{0}^{1212}\left(\varepsilon_{12}(t)-\int_{0}^{t} R_{G}(t-\tau) \varepsilon_{12}(\tau) d \tau\right) ; \\
& \tilde{\sigma}^{13}=C_{0}^{1212}\left(\varepsilon_{13}(t)-\int_{0}^{t} R_{G}(t-\tau) \varepsilon_{13}(\tau) d \tau\right) ; \\
& \tilde{\sigma}^{23}=C_{0}^{2323}\left(\varepsilon_{23}(t)-\int_{0}^{t} R_{E}(t-\tau) \varepsilon_{23}(\tau) d \tau\right) .
\end{aligned}
$$

\section{Development of a finite element model}

We use a variational approach in obtaining the basic relations of the finite element method to model the behavior of the material described by law (18). We consider the application of the Lagrange variational principle in solving a boundary value problem for a spatial structure. The potential energy $\mathrm{P}$ for the structure can be determined as follows:

$$
\Pi=W-A,
$$

where $W$ is the energy of elastic deformation of the structure, $A$ is the work of external forces acting on the structure.

After constructing a discrete model of the body, we can record the variation of potential energy:

$$
\delta \Pi=\sum_{m=1}^{N} \delta W^{(m)}-\delta A .
$$

To construct a finite element stiffness matrix for a composite material, we consider separately the variation of the elastic deformation energy of a finite element $\delta W^{(m)}$ occupying volume $V^{(m)}$ :

$$
\delta W^{(m)}=\iint_{V^{(m)}} \tilde{\sigma}^{i j} \delta \varepsilon_{i j} d V^{(m)}
$$

Using the relations for the law of state (18) of homogeneous transtropic material, we will have: 


$$
\delta W^{(m)}=\iiint_{V^{(m)}} C_{0}^{i k k l}\left(\varepsilon_{k l}(t)-\int_{0}^{t} R(t-\tau) \varepsilon_{k l}(\tau) d \tau\right) \delta \varepsilon_{i j} d V^{(m)},
$$

where $R(t-\tau)$ is the difference kernel, which takes the form $R_{G}(t-\tau)$ if the indices $(k, l)$ take the values $(1,2),(2,1),(1,3)$ or $(3,1)$, in all other cases - the form $R_{E}(t-\tau)$.

Accordingly, we have the variation of the energy of viscoelastic deformation of a homogeneous material, presented in the coordinate system of reinforcement $x_{i}(i=1,2,3)$. The procedure for developing a stiffness matrix of a single finite element is convenient to perform in the local coordinate system $x_{i^{\prime \prime}}(i "=1,2,3)$ associated with a finite element. Finite element coordinate system $x_{i "}$ and reinforcement coordinate system $x_{i}$ are transformed into each other by spatial rotation. To convert the components of the tensor of elastic constants from the coordinate system of reinforcement to the coordinate system of a finite element, we use the formula:

$$
C^{i " j " k " l "}=C^{i j k l} a_{i}^{i "} a_{j}^{j "} a_{k}^{k "} a_{l}^{l "},
$$

where $a_{i}^{i "}=\partial x_{i^{\prime \prime}} / \partial x_{i}$ are the components of the coordinate transformation tensor.

Given that the global coordinate matrix and the system of solving equations should be represented in the global Cartesian coordinate system $z_{i^{\prime}}\left(i^{\prime}=1,2,3\right)$, we write the variation of the viscoelastic deformation of a homogeneous material, using the transformation:

$$
C^{i^{\prime} j^{\prime} k^{\prime} l^{\prime}}=C^{i^{\prime \prime} j^{\prime \prime} k^{\prime \prime} l "} a_{i^{\prime \prime}}^{i^{\prime}} a_{j^{\prime \prime}}^{j^{\prime}} a_{k^{\prime \prime}}^{k^{\prime}} a_{l^{\prime \prime}}^{l^{\prime}}
$$

where $a_{i}^{i^{\prime}}=\partial z_{i^{\prime}} / \partial x_{i^{\prime \prime}}$ are the components of the coordinate transformation tensor. These components are easily found by approximating the coordinates of the points of a finite element given by the functions of the form:

$$
z_{i^{\prime}}=\sum_{L=1}^{L_{m}} z_{i^{\prime}}^{L} N_{L}\left(x_{1^{\prime \prime}}, x_{2^{\prime \prime},} x_{3^{\prime \prime}}\right),
$$


where $z_{i^{\prime}}^{L}$ is the coordinate of $L$-th node for $i^{\prime}$-th direction in the basic coordinate system, $N_{L}\left(x_{1^{\prime \prime}}, x_{2^{\prime \prime}}, x_{3^{\prime \prime}}\right)$ is the form function of $L$-th node in the coordinate system of a finite element.

As a result of these transformations we come to the variation of the energy of viscoelastic deformation of a homogeneous material in the presentations, shown by the components of the global coordinate system:

$$
\delta W^{(m)}=\iiint_{V} C_{0}^{i^{\prime} j^{\prime} k^{\prime} l^{\prime}}\left(\varepsilon_{k^{\prime} l^{\prime}}(t)-\int_{0}^{t} R(t-\tau) \varepsilon_{k^{\prime} l^{\prime}}(\tau) d \tau\right) \delta \varepsilon_{i^{\prime} j^{\prime}} d V^{(m)} .
$$

Taking into account the last expression, we write down the variation of the potential energy of the body:

$$
\delta \Pi=\sum_{m=1_{V}}^{N} \iiint_{(m)} C_{0}^{i^{\prime} j^{\prime} k^{\prime} l^{\prime}}\left(\varepsilon_{k^{\prime} l^{\prime}}(t)-\int_{0}^{t} R(t-\tau) \varepsilon_{k^{\prime} l^{\prime}}(\tau) d \tau\right) \delta \varepsilon_{i^{\prime} j^{\prime}} d V^{(m)}-\delta A .
$$

Variations in the action of forces acting on the body may include the work of distributed volumetric $P^{i^{\prime}}$ and surface forces $F^{i^{\prime}}$ :

$$
\delta A=\iiint_{V} P^{i^{\prime}} \delta u_{i} \cdot d V+\iint_{S} F^{i^{\prime}} \delta u_{i} \cdot d S, \iint
$$

Where $u_{i}$ is the body movement in the basic coordinate system.

Given that according to the Lagrange variational principle, the variation of the potential energy of the body should be zero, we will have the following relationship:

$$
\begin{aligned}
& \sum_{m=1}^{N} \iiint_{V} \int_{(m)} C_{0}^{i^{\prime} j^{\prime} k^{\prime} l^{\prime}}\left(\varepsilon_{k^{\prime} l^{\prime}}(t)-\int_{0}^{t} R(t-\tau) \varepsilon_{k^{\prime} l^{\prime}}(\tau) d \tau\right) \delta \varepsilon_{i^{\prime} j^{\prime}} d V^{(m)}- \\
& -\iiint_{V} P^{i} \delta u_{i} d V-\iint_{S} F^{i^{\prime}} \delta u_{i^{\prime}} d s=0, \iint
\end{aligned}
$$


or after conversion

$$
\begin{gathered}
\sum_{m=1}^{N} \iint_{V^{(m)}} C_{0}^{i j^{\prime} k^{\prime \prime}{ }^{\prime}} \varepsilon_{k^{\prime} l^{\prime}}(t) \delta \varepsilon_{i j^{\prime}} d V^{(m)}- \\
-\sum_{m=1}^{N} \iiint_{V} C_{0}^{i j^{\prime} k^{\prime} l^{\prime}}\left(\int_{0}^{t} R(t-\tau) \varepsilon_{k^{\prime} l^{\prime}}(\tau) d \tau\right) \delta \varepsilon_{i^{\prime} j^{\prime}} d V^{(m)}- \\
-\iiint_{V} P^{i} \delta u_{i}^{i} d V-\iint_{S} F^{i^{\prime}} \delta u_{i^{\prime}} d s=0,
\end{gathered}
$$

Let us break the gap $[0, t]$ with a certain interval on $n$ parts. Assuming moving on each time interval $\left[t_{l}, t_{l+1}\right]$ changes linearly, the integral operator in (29) can be represented as follows:

$$
\begin{gathered}
\sum_{m=1}^{N} \iiint_{V^{(m)}} C_{0}^{i j^{\prime} k^{\prime} l^{\prime}} \varepsilon_{k^{\prime \prime} l^{\prime}}(t) \delta \varepsilon_{i^{\prime} j^{\prime}} d V^{(m)}- \\
-\sum_{m=1}^{N} \iiint_{I^{\prime m)}} C_{0}^{i^{\prime} k^{\prime} k^{\prime} l^{\prime}}\left(\sum_{l=0}^{n-1} \varepsilon_{k^{\prime} l^{\prime}}\left(t_{l}\right) \int_{t_{l}}^{t_{t+1}} R(t-\tau) d \tau\right) \delta \varepsilon_{i^{\prime} j^{\prime}} d V^{(m)}- \\
-\iiint_{V} P^{i^{\prime}} \delta u_{i^{\prime}} d V-\iint_{S} F^{i^{i}} \delta u_{i^{\prime}} d s=0,
\end{gathered}
$$

The first expression in the left-hand side of the equation is the variation of the energy of elastic deformation at a certain point in time, it serves as a basis for constructing a stiffness matrix of a finite element $\left\lfloor K^{n_{1} m_{1}}\right\rfloor$ when modeling the elastic deformation of the body:

$$
K^{n_{1} m_{1}} u_{n_{1}}(t) \delta u_{m_{1}}=\sum_{m=1}^{N} \iiint_{V^{(m)}} C_{0}^{i j^{\prime} j^{\prime} k^{\prime}{ }^{\prime}} \varepsilon_{k^{\prime} l^{\prime}}(t) \delta \varepsilon_{i^{\prime} j^{\prime}} d V^{(m)} .
$$

The second term in equation (30) is the hereditary part of the timedependent stiffness matrix, written as follows: 


$$
\begin{aligned}
& \sum_{l=0}^{n-1} R_{l} K^{n_{1} m_{1}}\left(t_{l}\right) u_{n_{1}}\left(t_{l}\right) \delta u_{m_{1}}= \\
& =\sum_{m=1}^{N} \iiint_{V^{(m)}} C_{0}^{i i^{\prime} k^{\prime} l^{\prime}}\left(\sum_{l=0}^{n-1} \varepsilon_{k^{\prime} l^{\prime}}\left(t_{l}\right) \int_{t_{l}}^{t_{t+1}} R(t-\tau) d \tau\right) \delta \varepsilon_{i^{\prime} j^{\prime}} d V^{(m)},
\end{aligned}
$$

where

$$
\tilde{R}_{l}=\int_{t_{l}}^{t_{l+1}} R(t-\tau) d \tau .
$$

Variation of volume and surface forces can also be reduced to nodal values:

$$
F^{m_{1}} \delta u_{m_{1}}=\iiint_{V} P^{i^{\prime}} \delta u_{i^{\prime}} d V-\iint_{S} F^{i^{\prime}} \delta u_{i^{\prime}} d s
$$

Taking into account the selected parts, the relationship (30) can be written as follows:

$$
\left[K^{n_{1} m_{1}} u_{n_{1}}\left(t_{n}\right)-\sum_{l=0}^{n-1} \dot{R}_{l} K^{n_{1} m_{1}}\left(t_{l}\right) u_{n_{1}}\left(t_{l}\right)-F^{m_{1}}\right] \delta u_{m_{1}}=0 .
$$

Taking into account that the variation of movements cannot be equal to zero, the expression in parentheses must be equal to zero, whence we obtain a system of solving equations for a homogeneous transtropic body modeling a fibrous composite material with a viscoelastic isotropic matrix and an elastic transtropic fiber:

$$
K^{n_{1} m_{1}} u_{n_{1}}\left(t_{n}\right)=\sum_{l=0}^{n-1} \check{R}_{l} K^{n_{1} m_{1}}\left(t_{l}\right) u_{n_{1}}\left(t_{l}\right)+F^{m_{1}}
$$

The first term in the right-hand side of the obtained system (35) is an additional load that simulates the viscoelastic properties of a homogeneous transtropic material.

\section{CONCLUSIONS}

The obtained equation (35) is a linearized system of algebraic equations, the solution of which in the first iteration gives an elastic solution for a homogeneous transtropic material. Performing further iterations makes it 
possible to obtain a stress-strain state step by step in the following moments of time. The properties of the components of the fibrous composite are laid down in the stiffness matrices in the right and left-hand sides of the equality. The rheological properties of the matrix material take into account the value $\breve{R}_{l}$, which is the integral of the difference kernel for a certain period of time. The main mathematical difficulty in the practical implementation of equation (35) is to find the original of the relaxation kernels from its image for certain types of difference kernels.

\section{SUMMARY}

An approach for determining the components of the stress-strain state of a viscoelastic transtropic composite is proposed. Its structural elements are a viscoelastic isotropic matrix and a perfectly elastic transtropic fiber. The viscoelastic properties of the material are described by the relations of the Boltzmann-Volterra hereditary theory. An integral Laplace transform has been used to homogenize the composite. After finding the temporal viscoelastic characteristics of the composite, the equation of state has been found. The Lagrange variational principle has been used to obtain the basic relations of the finite element method. The stiffness matrix and the basic solving equations of the finite element method for spatial structures made of composite have been developed.

\section{REFERENCES}

1. S. Sun, G. Sun, X. Du and J. Wu, "Bending of a Viscoelastic Beam Reinforced with Shape Memory Alloy Fibers", Journal of Shanghai Jiaotong University, 2002, 36 (11), pp. 1663-1666.

2. K. Sasaki, N. Saito, S. Amagi and T. Tsunoda, "Numerical analysis of residual stress in resin-molded products (2nd report, orthotropic visco-elastic analysis of residual stress in FRP structures)", Transactions of the Japan Society of Mechanical Engineers, Part A, 2000, 66 (652), pp. 2122-2127.

3. H. Yang and X. Guo, "Perturbation boundary-finite element combined method for solving the linear creep problem", International Journal Solids and Structures, 2000, Vol. 37, Issue 15, pp. 2167-2183.

4. S. N. Grebenyuk and E. L. Mizernaya, "Stress-strain state of fibrous composite materials under conditions of viscoelastic deformation", Visnik of the Volodymyr Dahl East Ukrainian National University, 2010, N 10 (152), Vol. 2. pp. 41-48.

5. V. G. Martynenko and G. I. Lvov, "The numerical technique for prediction of anisotropic viscoelastic properties of orthogonally fiber reinforced composite material", Bulletin of the National technical 
university "Kharkiv polytechnic institute". Collected Works. Series: Dynamics and Strength of Machines, 2016, N 46 (1218). pp. 44-51.

6. M. I. Klymenko, S. M. Grebenyuk and S. I. Homeniuk, Effective mechanical characteristics of the viscoelastic composites, Kherson: Helvetica, 2019.

\section{Information about the authors:} Grebenyuk S. M., Doctor of Technical Sciences, Head of the Department of Fundamental Mathematics,

Zaporizhzhia National University 66, Zhukovskoho str., Zaporizhzhia, 69600, Ukraine

Klymenko M. I.,

$\mathrm{PhD}$ in Physics and Mathematics, Associate Professor at the Department of Fundamental Mathematics,

Zaporizhzhia National University 66, Zhukovskoho str., Zaporizhzhia, 69600, Ukraine 